\title{
Focusing X-Ray Beams to Nanometer Dimensions
}

\author{
C. Bergemann, ${ }^{1, *}$ H. Keymeulen, ${ }^{2}$ and J. F. van der Veen ${ }^{2}$ \\ ${ }^{1}$ Laboratorium für Festkörperphysik, ETH-Hönggerberg, CH-8093 Zürich, Switzerland \\ ${ }^{2}$ Paul Scherrer Institut, CH-5232 Villigen, Switzerland and ETH-Zürich, Zürich, Switzerland
}

(Received 27 June 2003; published 10 November 2003)

\begin{abstract}
We address the question: what is the smallest spot size to which an x-ray beam can be focused? We show that confinement of the beam within a narrowly tapered waveguide leads to a theoretical minimum beam size of the order of $10 \mathrm{~nm}$ (FWHM), the exact value depending only on the electron density of the confining material. This limit appears to apply to all $\mathrm{x}$-ray focusing devices. Mode mixing and interference can help to achieve this spot size without the need for ultrasmall apertures.
\end{abstract}

DOI: 10.1103/PhysRevLett.91.204801

PACS numbers: 41.50.+h, 07.85.Qe, 61.10.-i

At synchrotron radiation sources a variety of focusing devices for hard $\mathrm{x}$ rays $(>8 \mathrm{keV})$ are presently in use for $\mathrm{x}$-ray diffraction, microscopy, and holography studies of small objects. Refractive lenses [1] and Fresnel zone plates [2] now produce beam sizes down to about $100 \mathrm{~nm}$, and Kirkpatrick-Baez mirror pairs can compress beams down to similar dimensions [3]. Confinement of $\mathrm{x}$-ray beams in gaps down to $40 \mathrm{~nm}$ has been achieved by use of planar waveguides, into which the beam was efficiently coupled either by use of a resonant beam coupler [4,5] or a Fresnel zone plate lens [6]. The beam may also be collected by a hollow glass capillary, having a wide entrance opening and tapering down to a small exit diameter [7].

Traditionally, the confinement of $x$ rays through tapered capillaries has been described using a simple ray optics approach. However, a full wave optics treatment is warranted whenever such devices are to deliver beam sizes in the submicron range. In this Letter, we present a general wave optics description of the beam propagation of $\mathrm{x}$ rays through waveguides. We demonstrate that there is a universal, wavelength-independent lower limit to the spot size that waveguide devices can focus to, owing to the small deviation of the index of refraction from unity, $n=1-\delta$, in the $\mathrm{x}$-ray regime. We argue that the same lower limit applies to all x-ray diffractive optics.

We treat the two conceptually simplest focusing devices in more detail: a wedge between two flat plates and a tapered circular capillary, which focus the x-ray beam in one and two dimensions, respectively. In general, we show that such devices act as a rigorous low-pass mode filter. The modes present at the downstream end may interfere such that a narrow focus with enhanced intensity results.

We first consider the planar waveguide geometry with an air gap as shown in Fig. 1(a); see also Ref. [8]. The upper plate, which is connected to a nanopositioning device, is slightly tilted, thus forming a wedge with the lower plate. In our experimental setup the wedge has an angle $\phi_{0}=7.71 \times 10^{-5} \mathrm{rad}$, a length of $4.85 \mathrm{~mm}$, and gap widths of $W_{1}=538 \mathrm{~nm}$ and $W_{2}=164 \mathrm{~nm}$ at its entrance and exit, respectively. The propagation of electromagnetic radiation through the wedge is described by the Helmholtz equation

$$
\Delta u+n^{2} k^{2} u=0,
$$

with $\Delta$ the Laplace operator, $k$ the wave vector, and $u$ the scalar wave field which represents the component $E_{z}$ of the electric field $\mathbf{E}=\left(0,0, E_{z}\right)$ perpendicular to the plane of Fig. 1.

The eigenmodes of Eq. (1) inside the wedge are readily solved in polar coordinates $(r, \phi)$ : The wedge is bounded by $\phi=0$ and $\phi_{0}$ [Fig. 1(a)], and we assume for now that the field does not penetrate into the confining plate material, i.e., approximately $u=0$ at this boundary. We find for the $m$ th eigenmode

$$
u_{m}(r, \phi)=H_{m \pi / \phi_{0}}^{(1)}(k r) \sin \left(\frac{m \pi \phi}{\phi_{0}}\right),
$$

where $H_{m \pi / \phi_{0}}^{(1)}$ is the Hankel function of the first kind of (fractional) order $m \pi / \phi_{0}$. Because the wedge angle is very small, this order is exceedingly large, $\sim 10^{5}$. The argument is even larger, $k r \sim 10^{8}$, so that $H_{m \pi / \phi_{0}}^{(1)}$ in Eq. (2) can be replaced by the asymptotic expansion [9]

$$
\sqrt{\frac{2}{\pi k r}} \exp i\left[k r-\frac{m \pi^{2}}{2 \phi_{0}}-\frac{\pi}{4}+\frac{m^{2} \pi^{2}}{2 \phi_{0}^{2} k r}\right] \text {. }
$$

(a)

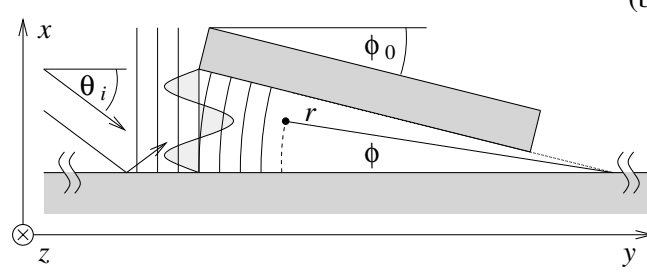

(b)

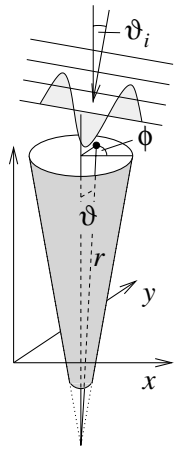

FIG. 1. Tapered waveguide geometries: (a) planar wedge and (b) circular capillary. Angles and distances are not to scale. 
Clearly, the beam gets focused as it travels along the wedge, with a corresponding intensity enhancement reciprocal to $r$.

In reality, the field in the confining material is nonzero, since the refractive index $n=1-\delta$ inside the wedge deviates only slightly from unity (e.g., for $\mathrm{SiO}_{2}, \delta=$ $2.58 \times 10^{-6}$ at the wavelength $\lambda=0.093 \mathrm{~nm}$ considered here). While we so far implicitly assumed total reflection from the waveguide walls, we now have to consider the extremely small critical angle $\theta_{c}=\sqrt{2 \delta}$. From the phase in Eq. (3), the effective local radial wave vector is $k(1-$ $m^{2} \pi^{2} / 2 \phi_{0}^{2} k^{2} r^{2}$ ); when this becomes comparable to the wave vector $k(1-\delta)$ in the medium, the waves start to leak out of the waveguide, preventing further focusing. This happens at $m$ times the critical gap width

$$
W_{c}=\frac{\lambda}{2 \theta_{c}},
$$

and we see below that this limit is quite universal.

We note [10] that $\delta=\lambda^{2} r_{0} n_{e} / 2 \pi$, with $r_{0}=2.818 \times$ $10^{-15} \mathrm{~m}$ the electron radius and $n_{e}$ the electron density of the material. $W_{c}$ is therefore independent of the wavelength $\lambda$ and rather weakly material dependent: as a good approximation we have $W_{c} \simeq 30 \mathrm{~nm} / \sqrt{\rho}$, where $\rho$ is the density of the waveguide material in $\mathrm{g} / \mathrm{cm}^{3}$. For $\mathrm{SiO}_{2}$, we find $W_{c}=21 \mathrm{~nm}$, while cladding the $\mathrm{SiO}_{2}$ surface with, e.g., a layer of $\mathrm{Au}$ would reduce $W_{c}$ to $8 \mathrm{~nm}$.

For a more general waveguide, we can recognize $\sim W_{c}$ as the minimum focus size from an analogy with the uncertainty principle in quantum mechanics: for a beam propagating along the waveguide, we can take $u$ to have the form $u=\psi e^{-i k y}$ with a slowly varying envelope function $\psi(x, y)$. Neglecting the small term $\partial^{2} \psi / \partial y^{2}$, we derive from Eq. (1) the beam propagation equation

$$
-\frac{1}{2 k^{2}} \frac{\partial^{2} \psi}{\partial x^{2}}+\delta \cdot \psi=\frac{i}{k} \frac{\partial \psi}{\partial y},
$$

which we recognize as the time-dependent Schrödinger equation, with $t \equiv(-) y, \hbar \equiv k^{-1}, m=1$, and $V \equiv \delta$.

The task of focusing an $\mathrm{x}$-ray beam is therefore equivalent to that of confining a quantum wave function inside a potential well of maximum height $V_{0} \equiv \delta$. For a bound state, the particle momentum is then limited by $p^{2} / 2 m<$ $V_{0}$; i.e., it has a maximum uncertainty $\Delta p \leq \sqrt{2 m V_{0}}$. The Heisenberg uncertainty principle now sets a lower bound for the position uncertainty $\Delta x \geq \hbar / \Delta p \geq \hbar / \sqrt{2 m V_{0}} \equiv$ $1 / k \sqrt{2 \delta}=W_{c} / \pi$, demonstrating that $W_{c}$ sets the scale for a universal lower limit to the beam size.

More specifically, our wedge geometry corresponds in the "quantum analog" to a potential well with constant height $\delta$ but shrinking width $W$. The eigenfunctions of such finite wells are studied in great detail in quantum mechanics textbooks [11]: the well is able to support $\left\lceil W / W_{c}\right\rceil$ bound states where $\lceil s\rceil$ is $s$ rounded up to the nearest integer. Inside the well the wave function is sinu- soidal; outside it decays exponentially. The decay length diverges when the well becomes too narrow to support the wave function. The lowest mode remains bound down to zero gap width, but its decay length in the material diverges for $W \rightarrow 0$, causing its full width at half (intensity) maximum (FWHM) to increase for gap widths smaller than $\sim W_{c}$. Numerically, the minimum achievable FWHM is $0.64 W_{c}$, in line with our general statement above, and is taken on at $W=0.44 W_{c}$. The situation remains similar in the presence of absorption $(\delta \rightarrow \delta+$ $i \beta$ complex), so that even pinhole apertures cannot beat the limit $W_{c}$.

The time evolution of the confined wave functions can be treated within the adiabatic approximation, but it is generally more useful to numerically solve Eq. (5) directly [8]. Figure 2(a) shows the results of such a calculation for $\mathrm{a} \mathrm{SiO}_{2}$ wedge, when the incoming beam consists of only the lowest mode: the beam gets focused down to a FWHM spot size of $13 \mathrm{~nm} \simeq 0.64 W_{c}$ and then diverges. (The calculation also takes account of absorption, with $\beta=1.23 \times 10^{-8}$ for $\mathrm{SiO}_{2}$ at the wavelength $\lambda=$ $0.093 \mathrm{~nm}$.)

Generally, the incident wave $u_{i}$ excites several modes $m$ in the waveguide, and the mode distribution is found by matching the amplitude of the incoming wave field to that of a linear combination of eigenmodes, $\sum A_{m} u_{m}$. In our setup, a prereflection of the incoming plane wave from the lower extended waveguide surface (Fig. 1) results in a field at the entrance that already has a standing-wave character similar to the eigenmodes of the wedge, so the incoming wave field has the form $u_{i}(x, y)=$ $\sin \left(k x \sin \theta_{i}\right) \exp \left(-i k y \cos \theta_{i}\right)$, where $\theta_{i}$ is the incidence angle of the plane wave with respect to the plane of the lower surface.

In cylindrical coordinates we then have $u_{i}(r, \phi) \simeq$ $\sin \left(k r \phi \theta_{i}\right) \exp \left[i k r\left(1-\theta_{i}^{2} / 2-\phi^{2} / 2\right)\right]$. Matching this to the eigenmodes [Eqs. (2) and (3)] at the entrance $r \equiv r_{i}$, we obtain the overlap integrals

$$
\begin{aligned}
A_{m}= & \sqrt{\frac{2 \pi k r_{i}}{\phi_{0}^{2}}} \exp i\left[\frac{\pi}{4}-\frac{k r_{i} \theta_{i}^{2}}{2}+\frac{m \pi^{2}}{2 \phi_{0}}-\frac{m^{2} \pi^{2}}{2 \phi_{0}^{2} k r_{i}}\right] \\
& \times \int_{0}^{\phi_{0}} d \phi \sin \left(k r_{i} \phi \theta_{i}\right) \sin \frac{m \pi \phi}{\phi_{0}} \exp \frac{-i k r_{i} \phi^{2}}{2}
\end{aligned}
$$

under the assumption that evanescent wave effects are negligible at the wide entrance opening. Optimal matching at the entrance is achieved if one of the nodes of the standing wave coincides with the position of the upper surface, i.e., if $\theta_{i}=\pi m /\left(\phi_{0} k r_{i}\right)$, with integer $m$. The incident field then couples most strongly to the eigenmode $m$. However, the phase factor $\exp \left(-i k r_{i} \phi^{2} / 2\right)$ in Eq. (6), which represents the phase mismatch between the cylindrically curved wave fronts of the wedge eigenmodes and the plane incident wave fronts, causes other eigenmodes to be excited as well. 
The magnitude of phase mismatch at the wedge entrance can be expressed by the parameter $\xi \equiv$ $\sqrt{k r_{i} \phi_{0}^{2} / \pi}$. Figures 2(b)-2(d) show the field intensities within the wedge for increasing $\xi$, for a $\mathrm{TE}_{2}(m=3)$ input mode [12], demonstrating how increasing $\xi$ leads to mode mixing and interference. Of particular interest is the experimental situation in Fig. 2(c), $\xi \simeq 1$, where the few excited modes are interfering to form a pattern of well-localized strong maxima. For example, the circled maximum exhibits an intensity enhancement of a factor of 6 relative to the maximum of the incident field, which is almost twice the factor expected from geometrical compression $\left(r_{i} / r \simeq 3.5\right)$. Its FWHM is only $\sim 20 \mathrm{~nm}$ wide, although the exit gap is much wider at $150 \mathrm{~nm}$. Interference may therefore be exploited to achieve a small $\mathrm{x}$-ray spot without having to produce an awkwardly small exit gap (but it cannot beat the universal limit of $\sim W_{c}$ ).

For higher input modes $m$, the influence of the evanescent waves becomes more significant since the critical gap width $m W_{c}$ is much larger. Figure 2(e) shows the field intensity when a virtually pure large- $m$ wedge eigenmode is excited at the entrance: essentially all radiation intensity disappears out of the gap at $W \simeq(m-1) W_{c}(\simeq$ $150 \mathrm{~nm}$ for the case shown, $m=8$ ) and is absorbed into the $\mathrm{SiO}_{2}$ plates. The transmission for this mode is correspondingly low. The wedge therefore acts as a rigorous low-pass mode filter, e.g., for incoherent (i.e., multimode) radiation.
The field profile across the exit plane of the wedge gives rise to a distinct far-field diffraction pattern, and this is how the predicted beam evolution can be verified. The diffracted intensity distribution $I\left(\theta_{i}, \theta_{e}\right)$ has been measured as a function of $\theta_{i}$ and the exit angle $\theta_{e}$, as described in Ref. [13]. The diffraction includes a postreflection of the exiting waves from the extended lower plate [8]. Figure 3(a) shows the measured distribution. Diffraction patterns have been calculated for $\xi=$ $0.30,0.94$, and 3.00 , and only the one at $\xi=0.94$ shows a striking resemblance to the experimental one. This demonstrates the sensitivity of the diffraction features to changes in the beam, and it experimentally verifies the validity of our numerical approach.

The theory of the planar wedge can readily be extended to the tapered circular capillary. Considering the capillary to be a conical cut out of a sphere [Fig. 1(b)] and writing the Helmholtz equation in spherical coordinates, one finds for its eigenmodes

$$
u_{l m}(r, \vartheta, \phi)=h_{l}^{(1)}(k r) l^{-m} J_{m}(l \vartheta) \cos (m \phi),
$$

with $h_{l}^{(1)}$ the spherical Hankel function of the first kind and $J_{m}$ the Bessel function [9]. Here, $l=j_{m s} / \vartheta_{0}$, with $j_{m s}$ the $s$ th zero of the Bessel function of $m$ th order and $\vartheta_{0}$ the half angle of the cone. Mode $(m, s)$ starts to leak out at a critical diameter $\sim j_{m s} \lambda / \pi \theta_{c}$. For the lowest mode $(0,1)$, this is $1.53 W_{c} \simeq 46 \mathrm{~nm} / \sqrt{\rho}$, and the spot reaches its minimum diameter (FWHM) of $0.74 W_{c}$ at a capillary
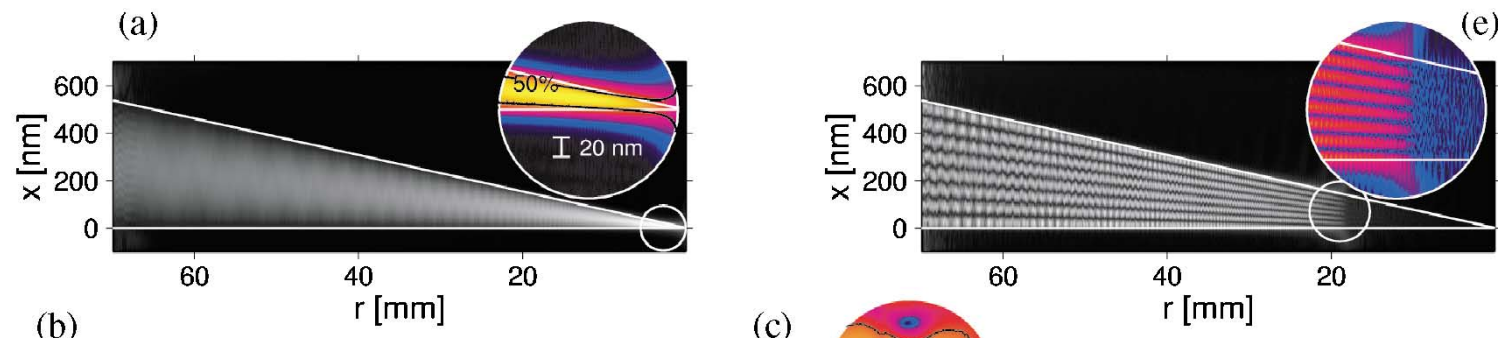

(e)

(c)

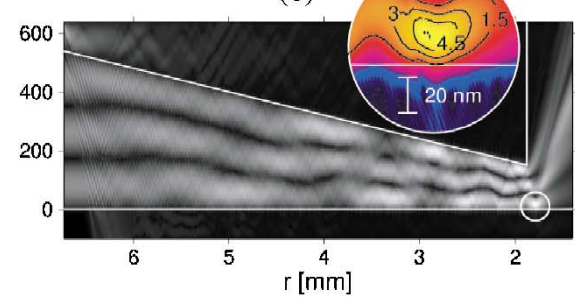

(d)

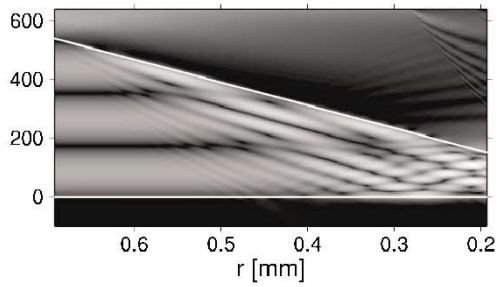

FIG. 2 (color). Calculated $\mathrm{x}$-ray intensity distribution inside a planar $\mathrm{SiO}_{2}$ wedge, with $\lambda=0.093 \mathrm{~nm}$ and with the incident standing wave matched to various input modes, for different values of the phase mismatch parameter $\xi$. The gray scale is linear in amplitude (not intensity); the magnification insets use a different color scheme for clarity. Note that the $x$ axes have identical scales, but the $r$ axes have not. (a) Evolution of input mode $\mathrm{TE}_{0}$ at low mismatch $(\xi=0.3)$; the inset shows the contour of half the local ( $r$-dependent) intensity maximum, demonstrating that $\sim 13 \mathrm{~nm} \mathrm{FWHM} \mathrm{is} \mathrm{the} \mathrm{minimum} \mathrm{beam} \mathrm{size.} \mathrm{(b)}-(\mathrm{d})$ Configuration mimicking the experiment, with $W_{1}=538 \mathrm{~nm}, W_{2}=150 \mathrm{~nm}$, a TE 2 input mode, and $\phi_{0}$ adjusted to give (b) $\xi=0.30$; (c) $\xi=$ 0.96, close to the actual experimental geometry, with the inset demonstrating the appearance of an intensity maximum: peaking at $6 \times$ the incident intensity and much narrower $(\sim 20 \mathrm{~nm})$ than the exit width; (d) $\xi=3.0$. (e) Low-pass filter: the TE mode $($ with low mismatch, $\xi=0.3$ ) ceases to be transmitted and radiates out of the waveguide as $W$ falls below $7-8 W_{c}$. The rapid intensity oscillations are due to the additional mismatch at the upper plate between the incident sinusoidal wave and the wedge modes which have an exponential tail inside the $\mathrm{SiO}_{2}$. 
(a)

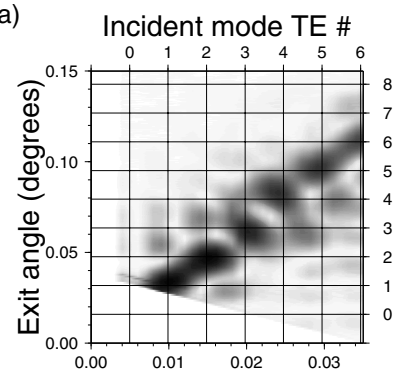

(c)

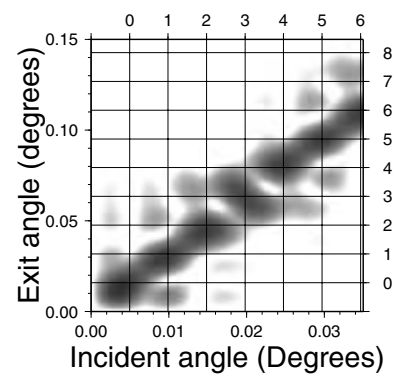

(b) Incident mode TE \#

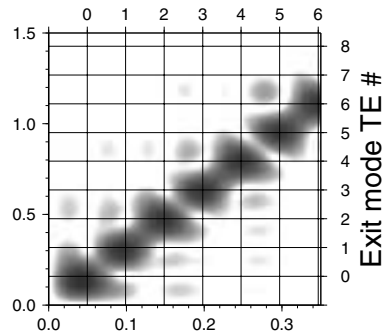

(d)

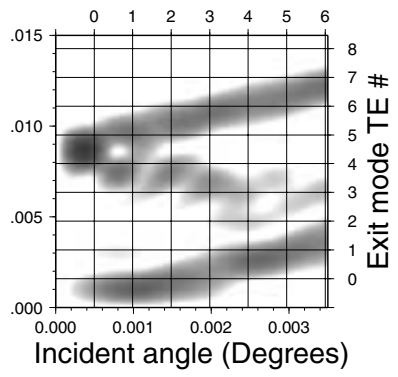

FIG. 3. Logarithmic density plots of the intensity $I\left(\theta_{i}, \theta_{e}\right)$ diffracted from the exit of a planar $\mathrm{SiO}_{2}$ wedge, with $\lambda=$ $0.093 \mathrm{~nm}$ : (b) -(d) are calculations for geometries similar to the respective panels of Fig. 2; (a) shows the measured intensity distribution, to be compared with (c), at $\xi=0.94$ (no data were taken for $\theta_{i}<0.004^{\circ}$ and the lower left corner of the plot). Note that the intensity distributions along the vertical lines labeled as incident mode \#2 are the diffracted fields for the $\mathrm{TE}_{2}$ input mode as shown in Figs. 2(b)-2(d).

diameter of $0.67 W_{c}$. Optimal phase matching of the incident beam to the lowest mode is achieved by prefocusing a Gaussian beam onto the entrance opening and aligning it with the cone axis. The analogy with the time-dependent Schrödinger equation still applies, but now with two spatial dimensions.

The beam size limit $\sim W_{c}$, derived here for waveguiding geometries, also applies to other $\mathrm{x}$-ray focusing devices. For example, the spot size achievable with a Fresnel zone plate is usually taken to be the outermost zone width $\Delta r$ for the main focus, and $\Delta r / n$ for $n$th order diffraction, i.e., a smaller spot size but at the cost of $1 / n^{2}$ efficiency. Volume gratings can increase the efficiency at higher orders [14,15], and so at first sight it appears that the spot size could be made arbitrarily small using small zone widths and/or high order diffraction. However, as we have seen, on exiting the zone plate the wave is effectively smeared out laterally over a length scale of $W_{c}$. This again sets a lower limit of $\sim W_{c}$ to the focal spot size, regardless of the order of diffraction. This is in line with the empirical spot size limit of $13 \mathrm{~nm}$ found in recent numerical studies [15] for zone plates made of nickel.

Note that Eq. (4) for $W_{c}$ is analogous to the expression $\lambda / 2 \mathcal{N} \mathcal{A}$ for the diffraction limited spatial resolution of

an optical lens system. Here, $\mathcal{N} \mathcal{A}$ is the numerical aperture in object space, which in our case equals $\theta_{c}$. It is the very small effective $\mathcal{N} \mathcal{A}$ of all x-ray optics that sets an ultimate limit to the spatial resolution of $\sim 10 \mathrm{~nm}$. Beam sizes down to $1 \mathrm{~nm}$, as desired for, e.g., single-atom $\mathrm{x}$-ray fluorescence experiments [16], therefore seem to be out of reach.

Nonetheless, a physical aperture of size $\sim W_{c}$ is still quite small and a challenge to make. However, our analysis of the focusing performance of a wedge-shaped waveguide demonstrated how mode mixing and interference can be exploited to achieve beam sizes down to near $W_{c}$ without the need for extremely small exit widths. In principle, such multimode interference effects can also be used to advantage in circular capillaries.

We are indebted to D. Bilderback for an inspiring discussion which led to this work. Discussions with M. J. Zwanenburg and A. Dolocan are gratefully acknowledged. The measurements were performed at beam line ID10 of the ESRF, Grenoble. This work was funded by the Swiss National Science Foundation (Project No. 2100-63738).

*Present address: Cavendish Laboratory, University of Cambridge, Madingley Road, Cambridge CB3 OHE, United Kingdom.

[1] B. Lengeler et al., Appl. Phys. Lett. 74, 3924 (1999).

[2] C. David, B. Nöhammer, and E. Ziegler, Appl. Phys. Lett. 79, 1088 (2002).

[3] K. Yamauchi et al., J. Synchrotron Radiat. 9, 313 (2002).

[4] Y. P. Feng et al., Phys. Rev. Lett. 71, 537 (1993).

[5] F. Pfeiffer et al., Science 297, 230 (2002).

[6] J. H. H. Bongaerts et al., J. Synchrotron Radiat. 9, 383 (2002).

[7] D. H. Bilderback, S. A. Hoffman, and D. J. Thiel, Science 263, 201 (1994).

[8] M. J. Zwanenburg et al., Phys. Rev. Lett. 82, 1696 (1999).

[9] M. Abramowitz and I. A. Stegun, Handbook of Mathematical Functions (Dover, New York, 1965), Chaps. 9.1-9.2.

[10] J. Als-Nielsen and D. McMorrow, Elements of Modern $X$-Ray Physics (Wiley, New York, 2001), p. 63.

[11] See, for example, F. Schwabl, Quantum Mechanics (Springer, New York, 1995), Chap. 3.4.

[12] The symbol $\mathrm{TE}_{n}$ stands for the transverse electric mode having $n$ nodes, excluding the ones at the boundaries.

[13] M. J. Zwanenburg et al., Physica (Amsterdam) 283B, 285 (2000).

[14] D. Hambach, G. Schneider, and E. M. Gullikson, Opt. Lett.. 26, 1200 (2001).

[15] A. N. Kurokhtin and A.V. Popov, J. Opt. Soc. Am. A 19, 315 (2002).

[16] D. H. Bilderback and R. Huang, AIP Series of Conference Proceedings, SRI Conference, San Francisco, 2003 (to be published). 